\title{
Vasoactive Intestinal Polypeptide Mediates Circadian Rhythms in Mammalian Olfactory Bulb and Olfaction
}

\author{
Jae-eun Kang Miller, ${ }^{1,2 \star}$ Daniel Granados-Fuentes, ${ }^{1 \star}$ Thomas Wang, ${ }^{1}$ Luciano Marpegan, ${ }^{1}$ Timothy E. Holy, ${ }^{2}$ \\ and Erik D. Herzog ${ }^{1}$ \\ Departments of ${ }^{1}$ Biology and ${ }^{2}$ Anatomy and Neurobiology, Washington University, St. Louis, Missouri 63130
}

\begin{abstract}
Accumulating evidence suggests that the olfactory bulbs (OBs) function as an independent circadian system regulating daily rhythms in olfactory performance. However, the cells and signals in the olfactory system that generate and coordinate these circadian rhythms are unknown. Using real-time imaging of gene expression, we found that the isolated olfactory epithelium and $\mathrm{OB}$, but not the piriform cortex, express similar, sustained circadian rhythms in PERIOD2 (PER2). In vivo, PER2 expression in the OB of mice is circadian, approximately doubling with a peak around subjective dusk. Furthermore, mice exhibit circadian rhythms in odor detection performance with a peak at approximately subjective dusk. We also found that circadian rhythms in gene expression and odor detection performance require vasoactive intestinal polypeptide (VIP) or its receptor VPAC2R. VIP is expressed, in a circadian manner, in interneurons in the external plexiform and periglomerular layers, whereas VPAC2R is expressed in mitral and external tufted cells in the OB. Together, these results indicate that VIP signaling modulates the output from the $\mathrm{OB}$ to maintain circadian rhythms in the mammalian olfactory system.
\end{abstract}

Key words: circadian; clock; olfaction; olfactory discrimination; rhythms; VIP

\section{Introduction}

Daily rhythms, including the sleep-wake and fasting-feeding cycles, have, over the past 40 years, been attributed to a circadian pacemaker in the mammalian suprachiasmatic nucleus (SCN). Recent discoveries that other cell types also exhibit autonomous circadian changes in gene expression highlighted the possibility that many tissues may regulate circadian changes in specific physiological and behavioral processes (Dibner et al., 2010; Welsh et al., 2010). The olfactory bulb (OB), for example, may function as an independent circadian system controlling daily changes in olfaction. OB slices exhibit intrinsic circadian rhythms in firing rate and PERIOD (PER) gene activity in vitro (Granados-Fuentes et al., 2004b). Mice show circadian rhythms in odor detection performance in the absence of time cues from the environment, and this circadian rhythm in olfactory sensitivity depends on the expression of canonical clock genes (Granados-Fuentes et al.,

Received Nov. 6, 2013; revised March 12, 2014; accepted March 24, 2014.

Author contributions: J.-e.K.M., D.G.-F., L.M., T.E.H., and E.D.H. designed research; J.-e.K.M., D.G.-F., T.W., and L.M. performed research; J.-e.K.M., D.G.-F., T.W., L.M., T.E.H., and E.D.H. analyzed data; J.-e.K.M., D.G.-F., T.E.H., and E.D.H. wrote the paper.

This work was supported by National Institute of Mental Health Grant 63104 (E.D.H.) and National Institutes of Health Director's Pioneer Award DP1 $0 D 006437$ and Grant R01 DC005964 (T.E.H.). We thank the members of the Holy and Herzog laboratories.

The authors declare no competing financial interests.

*J.-e.K.M. and D.G.-F. contributed equally to this work.

Correspondence should be addressed to Dr. Erik D. Herzog, Department Biology, Washington University, One Brookings Drive, Box 1137, St. Louis, M0 63130. E-mail: herzog@wustl.edu.

J.-e. K. Miller's present address: Department of Biological Sciences, Columbia University, 550 West 120 Street, Box 4822, New York, NY 10027.

L. Marpegan's present address: Department of Science and Technology, National University of Quilmes, Roque Sáenz Peña 352, Bernal, Buenos Aires, Argentina, B1876BXD.

DOI:10.1523/JNEUROSCI.4713-13.2014

Copyright $\odot 2014$ the authors $\quad 0270-6474 / 14 / 346040-07 \$ 15.00 / 0$
2011). Furthermore, daily rhythms in the OB and olfactory discrimination persist when circadian rhythms are eliminated in the SCN (Abraham et al., 2005) or when the SCN are ablated (Granados-Fuentes et al., 2004a). Thus, a circadian system that parallels the canonical SCN-dependent system appears to regulate olfaction. However, we do not know how olfactory circuits generate and coordinate daily rhythms in olfaction.

The neuropeptide vasoactive intestinal polypeptide (VIP) is produced by neurons sparsely distributed throughout the retina, neocortex, SCN, and OB (Shinohara et al., 1994, 1995; Crespo et al., 2002; Gracia-Llanes et al., 2003; Brand et al., 2005; Vosko et al., 2007; Hu et al., 2011; Nakamachi et al., 2012; Zou et al., 2014). Coordinated circadian oscillations in locomotion depend on VIP signaling among SCN cells. Loss of VIP or its cognate receptor VPAC2R (encoded by the Vipr2 gene) results in a loss of daily rhythms in running-wheel behavior and desynchronized rhythms in spontaneous firing and gene expression among $\mathrm{SCN}$ neurons (Harmar et al., 2002; Colwell et al., 2003; Aton and Herzog, 2005; Brown et al., 2005; Maywood et al., 2006). Here, we tested the hypothesis that VIP is required for circadian rhythms in the $\mathrm{OB}$ and in olfaction.

\section{Materials and Methods}

Animals and locomotor activity recordings. All procedures were in accordance with the guidelines of the Washington University Institutional Animal Care and Use Committee and National Institutes of Health. Male mice ranging in age from 8 to 12 weeks were maintained on a C57BL/6 genetic background and selectively crossed to produce heterozygous or homozygous alleles of the PER2::LUCIFERASE (LUC) knock-in gene (founders generously provided by Dr. J. Takahasi, University of Texas Southwestern, Dallas, TX), Vip gene deletion (founders generously provided by Drs. C. Colwell and J. Waschek, University of California, Los Angeles, Los Angeles, CA), Protocadherin-Cre;Ai38 transgene (RIKEN 
BioResource Center and The Jackson Laboratory), and Gad65-EGFP transgene (The Jackson Laboratory). Mice were maintained in a $12 \mathrm{~h}$ light/dark (LD) cycle or constant darkness (DD) in the Danforth campus or the medical campus animal facility at Washington University.

Locomotor activity and olfactory performance were recorded from mice housed individually in cages with ad libitum access to food, water, and a running wheel. Light level and wheel revolutions in $1 \mathrm{~min}$ bins were stored on a computer (Clocklab; Actimetrics). Animals were in LD (lights on at 7:00 A.M.) for $\sim 3$ weeks during the olfactory training sessions and then tested for olfactory discrimination after $2 \mathrm{~d}$ in DD as described previously (Granados-Fuentes et al., 2011). Zeitgeber times (ZT) 0 and 12 refer to light onset and offset in LD, respectively. Circadian time (CT) 12 refers to activity onset in DD when the mice began running $>15$ revolutions per minute for at least $6 \mathrm{~h}$ each day (Clocklab). Other CTs were calculated in circadian hours from CT 12 by dividing the freerunning period of locomotor activity by 24 .

Surgery. To record Per2 gene expression in the OB in vivo, we created a cranial window over the $\mathrm{OB}$. Mice were anesthetized with isoflurane ( $2.5 \%$ for induction and $1 \%$ for maintenance), warmed at $37^{\circ} \mathrm{C}$ on a heating pad, and treated with an ophthalmic ointment to protect the eyes. Sterile surgical procedures were used throughout. For the in vivo PER2::LUC imaging experiments, a 2.5 -mm-diameter craniotomy was created over the OB using a dental drill (Abraham et al., 2005) during subjective night. We fixed a glass coverslip (\#1 thickness, $3 \times 4 \mathrm{~mm}$ ) above the OB with dental acrylic and filled the space between the dura and the coverslip with $2 \%$ agarose in artificial CSF (in mM: $126 \mathrm{NaCl}, 26$ $\mathrm{NaHCO}_{3}, 3 \mathrm{KCl}, 1 \mathrm{NaH}_{2} \mathrm{PO}_{4}, 2 \mathrm{MgSo}_{4}, 2 \mathrm{CaCl}_{2}$, and $10 \mathrm{~mm}$ glucose). For systemic luciferin delivery, a mini-osmotic pump (model 2001; Alzet, Durect) filled with beetle D-luciferin $(30 \mathrm{mg} / \mathrm{ml}$ in $0.1 \mathrm{M} \mathrm{PBS,} \mathrm{pH} 7.2$; Promega) was implanted under the skin on the back. The pump released luciferin at a constant rate $(1 \mu \mathrm{l} / \mathrm{h})$ for 1 week.

In vivo bioluminescence imaging. We recorded bioluminescence from two genotypes of mice (Vip ${ }^{-/-} ;$PER2::LUC and $\operatorname{Vip}^{+/-} ;$PER2::LUC) maintained in either LD or DD. Up to four mice were simultaneously anesthetized and imaged (see Fig. 2A) with an ultrasensitive, intensified charged coupled device camera (XR/Mega-10Z) using a custom-built imaging system (Onyx Box; Stanford Photonics). Mice were returned to their home cages after each imaging session so that wheel running could be continuously monitored. We imaged each mouse for 10 min every $4 \mathrm{~h}$ for $60 \mathrm{~h}$. During the dark phases of their light cycle, mice were transferred under dim red illumination. After the imaging experiment, mice were killed with $\mathrm{CO}_{2}$ and rapidly decapitated. We cultured a subset of PER2::LUC OBs that had been imaged in vivo. All ( $n=9 \mathrm{Vip}^{-1-}$ and 7 $V_{i p^{+/-}}$) showed bioluminescence signals above background for at least $6 \mathrm{~d}$ in vitro, indicating they were healthy.

Tissue culture and in vitro bioluminescence measurements. Briefly, male PER2::LUC knock-in mice were killed with $\mathrm{CO}_{2}$ and decapitated. Their brains were quickly collected in chilled HBSS, pH 7.2 (Sigma), supplemented with 0.01 м HEPES (Sigma), $100 \mathrm{U} / \mathrm{ml}$ penicillin, $0.1 \mathrm{mg} / \mathrm{ml}$ streptomycin, and $4 \mathrm{~mm} \mathrm{NaHCO}_{3}$ (Invitrogen). Coronal brain sections $(250 \mu \mathrm{m})$ were obtained with a vibratome slicer (OTS-4000; Electron Microscopy Sciences). The olfactory epithelium (OE) was dissected from inside the nasal cavity. The pieces of the OB, piriform cortex (PC), and OE were cultured individually on a Millicell-CM membrane (Millipore) in a Petri dish with $1 \mathrm{ml}$ of DMEM (Sigma) supplemented with $10 \mathrm{~mm}$ HEPES (Sigma), $2.2 \mathrm{mg} / \mathrm{ml} \mathrm{NaHCO}_{3}$ (Invitrogen), and $0.1 \mathrm{~mm}$ beetle luciferin (Promega). Petri dishes were sealed with grease and placed under photomultiplier tubes ( $\mathrm{HC} 135-11 \mathrm{MOD}$; Hamamatsu) at $37^{\circ} \mathrm{C}$ in the dark. Bioluminescence was recorded in $6 \mathrm{~min}$ bins for at least $6 \mathrm{~d}$.

Immunofluorescence. Animals were anesthetized with $2.5 \%$ tribromoethanol (Avertin) and transcardially perfused with $0.9 \%$ saline, followed by chilled $4 \%$ paraformaldehyde (PFA), pH 7.2. Brains were kept in PFA for $24 \mathrm{~h}$ and then transferred to $30 \%$ sucrose in PBS, pH 7.2, for $3 \mathrm{~d}$ until the brains sunk. Brains were quickly frozen with 2-methylbutane (Sigma) and stored at $-80^{\circ} \mathrm{C}$. Coronal sections $(30 \mu \mathrm{m}$ thick) were obtained with a cryostat (CM1850; Leica) and stored as six sets in Watson's cryoprotectant solution, $\mathrm{pH} 7.2$, at $-20^{\circ} \mathrm{C}$. For antibody staining, coronal sections were blocked in $10 \%$ bovine serum albumin (BSA) and $0.25 \%$ Triton X-100 in PBS for 60 min at room temperature. Primary antibodies to olfactory marker protein (OMP; goat, 1:2000; catalog \#544-10001; Wako), VIP (rabbit, 1:2000; Immunostar), GFP (chicken, 1:500; Abcam), and VPAC2R (rabbit, 1:1000; Abcam) were applied in 3\% BSA and $0.25 \%$ Triton X-100 in PBS for overnight at $4^{\circ} \mathrm{C}$. Secondary antibodies ( $1: 200$ in $3 \%$ BSA and $0.25 \%$ Triton X-100 in PBS, donkey antibody to goat IgG conjugated with TRITC, donkey antibody to rabbit IgG conjugated with $\mathrm{Cy} 2$, or goat antibody to rabbit IgG conjugated with $\mathrm{Cy} 3$; Jackson ImmunoResearch) were applied for $2 \mathrm{~h}$ at room temperature. Cell nuclei were stained with DAPI. Images were taken with a confocal microscope (A1 confocal laser microscope system; Nikon Instruments).

DAB immunohistochemistry. Mice were perfused at four CTs (CT 6, 12, 18 , and $24 ; n=3-4$ mice per time point), and the brains were processed for immunolabeling of the OB. Briefly, coronal cryosections $(30 \mu \mathrm{m}$ thick) of the OB were incubated with a VIP antibody (rabbit anti-VIP, 1:2000; Immunostar) and then processed according to the avidin-biotin method (ABC kit, pk6101; Vector Laboratories). Digitized images (Retiga 1350EX; QImaging) using QImaging Software were taken using standardized illumination for all sections. The brightness and contrast of all images were processed identically with NIH ImageJ (http://rsbweb. nih.gov/ij). To quantify the intensity of expression, we averaged the pixel intensity of VIP-immunoreactive cell bodies in the external plexiform layer (EPL; 10 cell bodies per brain in two sections at a certain CT) of the $\mathrm{OB}$ and subtracted the average background intensity in the granule cell layer (GCL).

Olfactometry. We measured olfactory thresholds using previously published methods (Granados-Fuentes et al., 2011). Briefly, mice were trained in an olfactometer to sniff in a central nose cone and then poke their noses into a left or right nose cone to indicate whether they detected the presence of an odor. Mice worked for a reward (30 $\mu$ l of tap water) delivered to the lateral cones after correct responses. We tested olfactory discrimination for five calibrated vanilla (Durkee)/water dilutions (1: $10^{1}, 1: 10^{2}, 1: 10^{3}, 1: 10^{4}$, and $\left.1: 10^{5}\right)$ as a function of CT. All dilutions of vanilla were calibrated using a photoionization detector (ppbRAE3000; RAE Systems). For analysis, we used the first 125 trials from each mouse at each CT. Mice were tested under constant dim red light after $2 \mathrm{~d}$ and 2 weeks in DD. Time of testing was defined relative to the daily onset of locomotor activity (CT 12) for WT mice or for arrhythmic Vip ${ }^{-/-}$and Vipr $2^{-1-}$ mice as projected CT 12 , which was defined as the time of lights off (7:00 P.M.). Data were collected at $8 \mathrm{~h}$ intervals to ensure that mice were thirsty so that it took $48 \mathrm{~h}$ to collect data for six CTs. At the end of each testing session, mice returned to their home cages. Mice were tested in three groups started at different CTs to avoid effects attributable to testing sequence.

Data analysis. We fit each in vitro bioluminescence trace with a damped sine function (Lumicycle software; Actimetrics) and defined data with a goodness of fit $>80$ as rhythmic. The period of PER2 expression was calculated using Cosopt analysis. In vivo bioluminescence images were processed using NIH ImageJ. Image frames over $15 \mathrm{CT}$ points were aligned using an NIH ImageJ plug-in, and an ROI over the OB was manually drawn for each mouse. To evaluate bioluminescence traces over $2 \mathrm{~d}$ for rhythmicity, JTK-Cycle test (combination of JonckheereTerpstra test for monotonic ordering and Kendall's $\tau$ test) was used (Hughes et al., 2010). Briefly, this nonparametric test estimates the period, amplitude, and phase of rhythmic data based on the order of the data (e.g., monotonically increasing). We also used a one-way ANOVA for repeated measures and a Tukey's post hoc test for differences across time (Origin 8.0; OriginLab).

For olfactometry, we calculated the percentage of correct responses. The number of trials in which a mouse correctly discriminated the presentation of odor (hits) or air (correct rejections) divided by the total number of trials as calculated previously (Slotnick and Restrepo, 2005). We used a two-way ANOVA for repeated measures and a Tukey's post hoc test for differences across time (Origin 8.0; OriginLab).

\section{Results}

Intrinsic circadian rhythmicity in PER2 expression at early stages of the olfactory circuit

To determine how olfactory circuits generate and coordinate daily rhythms in olfaction, we first examined which parts of the 
olfactory circuit exhibit intrinsic circadian rhythms in PER2 expression. We recorded bioluminescence from isolated tissues for $6 \mathrm{~d}$ and found that both the $\mathrm{OE}$ (Fig. 1; period of $19.1 \pm 0.3 \mathrm{~h}$; goodness of fit, $92.7 \pm 2.5$, mean \pm SEM; $n=6$ slices $)$ and $\mathrm{OB}$ (period of $23.9 \pm 1.8 \mathrm{~h}$; goodness of fit, $94.1 \pm 3.4 ; n=6$ ) exhibited intrinsic circadian rhythms in PER2 expression, but the PC did not (goodness of fit, $60.9 \pm$ 16 ; $n=7)$. The OE cultures had significantly shorter circadian periods than the OB cultures ( $p<0.05$, Student's $t$ test). This finding suggests that circadian rhythms are intrinsic to the first two stages of olfactory processing.

\section{The OB exhibits a circadian rhythm in PER2 expression in vivo}

We next determined whether the mouse OB exhibits circadian oscillations in vivo. For this, we recorded bioluminescence every $4 \mathrm{~h}$ for $60 \mathrm{~h}$ through a cranial window from homozygous PER2::LUC knock-in mice maintained in DD for 2 weeks. The mice were briefly anesthetized during imaging $(\sim 20 \mathrm{~min})$ and then returned to their home cages (Fig. $2 A$ ). The OB exhibited reliable, high-amplitude circadian changes in bioluminescence with a twofold to fivefold increase each night, peaking at approximately CT 20 and with a period of $22.9 \pm 0.5 \mathrm{~h}$ (mean \pm SEM). These circadian rhythms were statistically significant in all imaged mice (Fig. 2B; JTK-Cycle test; $n=7$ mice). Pooling the data from all mice, we found that average PER2 expression in the OB differed between peaks and troughs (Fig. $2 D ; F_{(14,99)}=7.1,{ }^{\star} p<$ 0.05 and ${ }^{* *} p<0.005$, one-way ANOVA with a Tukey's post hoc test; $n=7$ mice). This in vivo finding supports our in vitro findings and suggests that an intrinsic circadian rhythm arises at early stages of the olfactory circuit.

\section{Localization of VIPergic neurons and VPAC2 receptors in the $\mathrm{OB}$}

Previous studies demonstrated that bilateral lesions in the SCN do not disrupt circadian rhythms of PER1 expression of the OB in rats or odor detection performance in mice (Granados-Fuentes et al., 2006). However, it is unknown how cells of the OB maintain coordinated circadian rhythms independent of the SCN. VIP, expressed by neurons in the SCN and OB (Sanides-Kohlrausch and Wahle, 1990; Gracia-Llanes et al., 2003), was shown to be required for synchronized circadian oscillations among cells in the SCN (Aton and Herzog, 2005; Aton et al., 2005). However, whether VIP serves a similar function in the OB has not been explored. Because boutons of VIP-expressing cells in the OB have been reported to appose mitral (MT)/external (ET) tufted cell bodies (Gracia-Llanes et al., 2003), we postulated that VIP modulates the output of the $\mathrm{OB}$ to regulate daily rhythms in olfaction. To examine this, we first examined expression patterns of VIP and its receptor VPAC2R in the mouse OB.

We found that VIP immunolabeling colocalized with fluorescence in Vip-cre/+;floxed-tdtomato/+ knock-in mice. Therefore, we used the fluorescent reporter to characterize the distribution of VIP cells in the OB. Consistent with previous studies in other species (Crespo et al., 2002; Gracia-Llanes et al., 2003), VIP was expressed within the somata of cells in the external plexiform layer (EPL; Fig. 3, Table 1; $50.1 \pm 0.08 \%$ of total VIP-positive cells in the field of view, mean \pm SEM; $n=5 \mathrm{sec}$ tions from 4 mice), the periglomerular layer (PGL; Table 1,
$48.5 \pm 0.1 \%$ of total VIP-positive cells in the field of view), and sparsely within the granule cell layer (GCL; Table $1 ; 1.4 \pm 0.004 \%$ of total VIP-positive cells in the field of view). Most VIP-positive cells within the EPL were GAD65 positive (Table 2; $76.1 \pm 5.9 \%$ ), whereas only small fractions were GAD65 positive within the PGL and GCL (Table 2; $9.8 \pm 3.9$ and $16.7 \pm 16.7 \%$, respectively). As a control, we examined VIP staining in the OB of $\mathrm{Vip}^{-1-}$ mice and found no staining in the EPL or GCL and a few positive cells $(2.5 \pm 0.5)$ per slice in the PGL $\left(n=2 \mathrm{Vip}^{-/-}\right.$mice $)$, indicating that a subset of neurons in the EPL, PGL, and GCL produce VIP in the OB.

VPAC2R was strongly expressed within the MT/ET cell layer, the principal projection cells in the OB (Fig. 3, Table 1; $87.7 \pm$ $2.5 \%$ of total VPAC2R-positive MT/ET cells in the field of view; $n=6$ slices from 4 mice). VPAC2R staining colocalized with a subset of protocadherin-GFP-expressing MT/ET cells and was not found in cell bodies in other layers of the OB. Because immunoreactivity for VPAC2R in the OB of Vipr $2^{-/-}$mice was negligible ( $n=2$ mice; $10 \pm 3$ VPAC2R-positive cells per OB; $7.3 \pm$ $2.2 \%$ of all VPAC2R-positive cells found in WT OB), we conclude the staining was specific to VPAC2R. VPAC2R expression was most intense in the cell bodies of MT/ET cells but also was present in their processes especially within the glomeruli. These findings suggest that local VIP signaling from a subset of neurons in the EPL and PGL primarily targets the dendrites and cell bodies of MT/ET cells, the output from the OB.

\section{A circadian rhythm of VIP expression in the OB}

We next explored whether VIP levels in the OB change with CT. We quantified the intensity of VIP immunolabeling in the somata of neurons of the EPL from mice killed at one of four times of day in DD. VIP expression in the cell bodies of EPL neurons approximately doubled from a minimum at approximately midday (CT 6) to a peak at approximately midnight (CT 18; Fig. $3 ; F_{(3,66)}=$ 3.3; $p<0.05$, one-way ANOVA with a Tukey's post hoc test). These results suggest that VIP is synthesized and/or released from EPL neurons in a circadian manner with a peak in expression during the middle of the night.

\section{VIP is required for a circadian rhythm in PER2 expression of the $\mathrm{OB}$ in the absence of external timing cues}

To test whether VIP is required for the circadian rhythm in PER2 expression in the $\mathrm{OB}$, we monitored in vivo circadian changes in the bioluminescence from the OB in VIP-deficient, PER2::LUC mice. In contrast to $V i p^{+/-}$mice, $V i p^{-/-}$mice exhibited weak or no circadian changes in PER2 after 2 weeks in DD (Fig. $4 ; n=6$ of 7 mice failed the JTK-Cycle test for rhythmicity) and a modest reduction in the peak-to-trough amplitude in PER2 expression $\left(2.9 \pm 0.3\right.$-fold for $V i p^{+/-}$vs $2.2 \pm 0.1$-fold for $V i p^{-/-}$, mean \pm SEM; $p<0.05$, Wilcoxon's rank-sum test). Pooling data from the VIP-deficient mice, we found that PER2 bioluminescence did not 
A
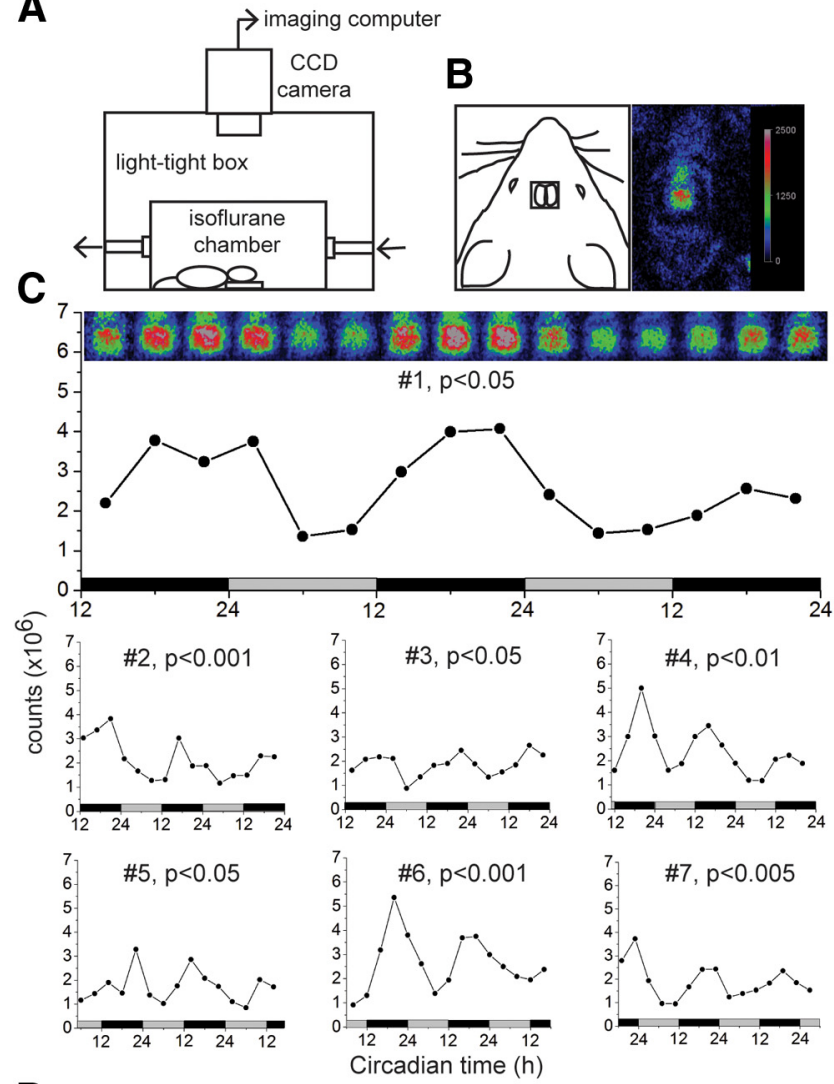

D

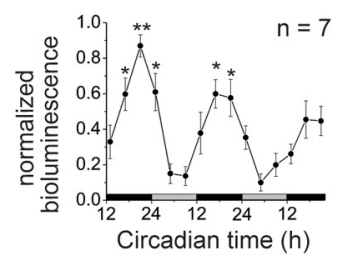

Figure 2. Circadian rhythms in PER2 expression from the $O B$ in vivo. $A$, $A$ drawing of the in vivo bioluminescence imaging setup. At $4 \mathrm{~h}$ intervals over $56 \mathrm{~h}, \mathrm{Vip}^{+/-} ;$; PER2::LUC mice were briefly anesthetized and transferred to a light-tight imaging box in which bioluminescence from the bilateral $O B s$ was imaged through a cranial window in $D D$. $B$, A drawing of a mouse positioned in the imaging box with a cranial window (left) and a pseudocolored image of its bioluminescent signal from $10 \mathrm{~min}$ exposures (right). C, Pseudocolored images of bioluminescence recorded every $4 \mathrm{~h}$ from the $0 B$ of mouse 1 after 2 weeks in $D D$ and bioluminescence levels show a daily rhythm peaking in the late subjective night (CT 20; top). PER2 expression was circadian in all seven mice with a twofold to fivefold increase from day to night. $\boldsymbol{D}$, Bioluminescence from each mouse was normalized (minimum is 0 and maximum is 1 ) and then averaged in $2 \mathrm{~h}$ bins across mice maintained in DD for 2 weeks. PER2 levels significantly differed from peak-to-trough ( ${ }^{*} p<0.05$ for $\mathrm{CT} 17$ and $\mathrm{CT} 1$ on the first imaging day and $\mathrm{CT} 17$ on the second day vs CT 5 and CT 9 on the first day and $C \mathrm{~T} 5$ on the second day; ${ }^{*} p<0.05$ for $\mathrm{CT} 21$ on the second day vs CT 5 and CT 9 on the first day; ${ }^{* *} p<0.005$ for CT 21 on the first day vs CT 13, CT 5 , and CT 9 on the first day and CT 5, СТ 9, and (T 13 on the second day; $n=7$ mice). С 24 denotes the daily offset of locomotion (subjective dawn), and CT 12 denotes the daily onset of locomotion (subjective dusk). Gray and black bars denote subjective light and dark times, respectively. Counts are the mean number of photons integrated over $10 \mathrm{~min}$.

differ with CT (Fig. $4 B ; F_{(14,84)}=2.8 ; p>0.5$, one-way ANOVA with a Tukey's post hoc test; $n=7$ mice). Thus, loss of VIP resulted in arrhythmic PER2 expression of the OB in $86 \%$ of the mice tested.

Interestingly, PER2 activity exhibited a daily rhythm in the $\mathrm{OB}$ of both control and Vip ${ }^{-1-}$ mice in LD (Fig. 5; $p<0.05$ for $V i p^{+/-}$and $p<0.05$ for Vip ${ }^{-1-}$, JTK-Cycle test; $n=7$ mice for both groups). Together, these results suggest that VIP is required
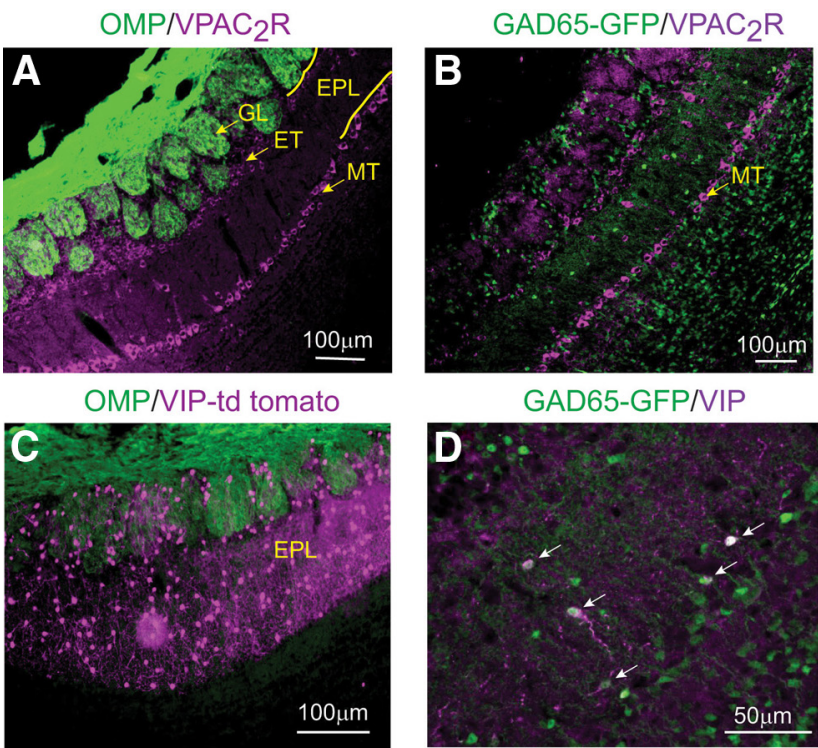

VIP-td tomato/VPAC $2 \mathrm{R}$

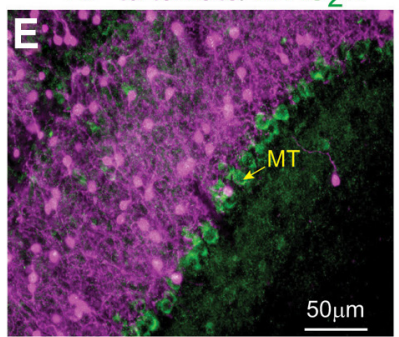

Pcadh21-GFP/NPAC 2 R
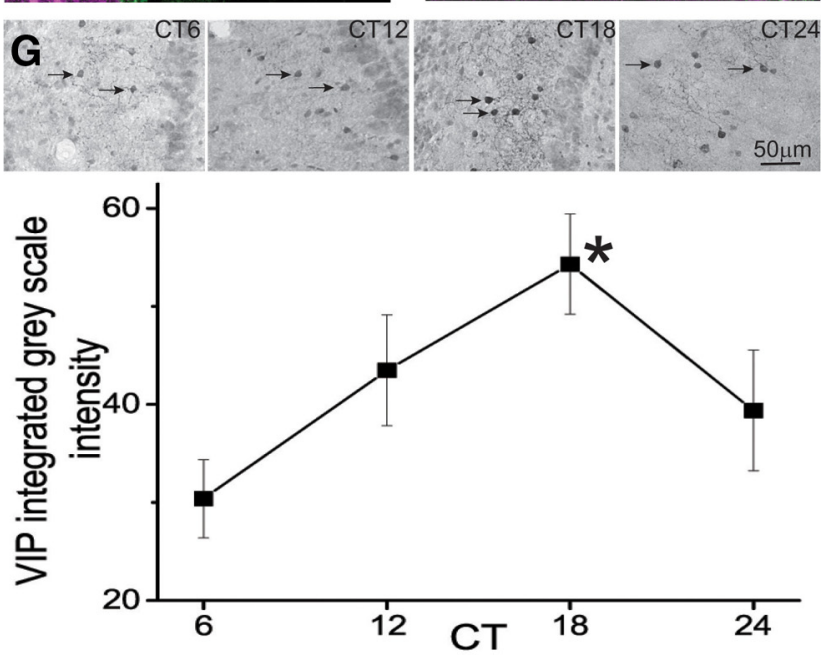

Figure 3. Both VIP and VPAC2R are expressed in the mouse $O B$. Representative confocal images of $O B$ sections stained with antibodies against VPAC2R (magenta) and OMP (green; $A$ ), and Vip;tdtomato (magenta) and OMP (green; $C$ ). VPAC2R staining was intense in the ET and MT cells (magenta, $\boldsymbol{A}, \boldsymbol{B}, \boldsymbol{F} ;$ green, $\boldsymbol{E}$ ). VIP staining or Vip;tdtomato expression was present mainly in the PGL and EPL (magenta, $(-E)$ ). OB sections of Gad65-EGFP mice stained with antibodies against VPAC2R (magenta) or VIP (magenta) revealed that a large fraction of VIP-positive neurons was GABAergic $(\boldsymbol{D})$, whereas none of VPAC2R-positive neurons were GABAergic $(\boldsymbol{B})$. Protocadherin-21 (Pcadh21) labeling for MT/ET cells $(\boldsymbol{F})$ shows that VAPC2R staining was specific to a subset of these neurons. Arrows in $\boldsymbol{D}$ and $\boldsymbol{F}$ indicate examples of cell bodies with both VIP staining and Gad65-EGFP expression and examples of MT/ET cells expressing VPAC2R, respectively. Representative neurons located mainly in the EPL immunolabeled for VIP (G, arrows) at four (Ts. The quantification of the integrated intensity of immunolabeled somata in the $\mathrm{OB}$ shows a circadian rhythm with a peak in VIP expression at $\mathrm{CT} 18 .{ }^{*} p<0.05$, CT 18 versus CT6. Mean \pm SEM. GL, Glomerular layer. 
Table 1. Distributions of VIP and VPAC2R expression in different regions of $O B$

\begin{tabular}{lllllr}
\hline & MT & ET & GCL & EPL & \multicolumn{1}{l}{ PGL } \\
\hline VPAC2R & $40.7 \pm 2.0 \%$ & $47.0 \pm 2.5 \%$ & $7.0 \pm 1.3 \%$ & $0 \%$ & $5.2 \pm 0.9 \%$ \\
VIP & $0 \%$ & $0 \%$ & $1.4 \pm 0.004 \%$ & $50.1 \pm 0.1 \%$ & $48.5 \pm 0.1 \%$ \\
\hline
\end{tabular}

The fraction of DAPI-positive cells that labeled for VIP or VPAC2R are reported. $n=3$ sections from each of two C57BL/ 6 mice and $n=4$ sections from two Gad65-EGFP mice for VIP staining; $n=3$ sections from two C57BL/6 mice and $n=3$ sections from two Gad65-EGFP mice for VPAC2R staining.

Table 2. The fraction (mean number of the coexpressing cells inside the parentheses) of VIP- or VPAC2R-positive neurons that coexpressed Gad65-EGFP in the $0 B$

\begin{tabular}{llllll}
\hline & MT & ET & GCL & EPL & PGL \\
\hline VPAC2R & $0 \%$ & $0 \%$ & $0 \%$ & $0 \%$ & $0 \%$ \\
VIP & $0 \%$ & $0 \%$ & $16.7 \pm 16.7 \%(0.6)$ & $76.1 \pm 5.9 \%(11.5)$ & $9.8 \pm 3.9 \%(0.9)$ \\
\hline
\end{tabular}

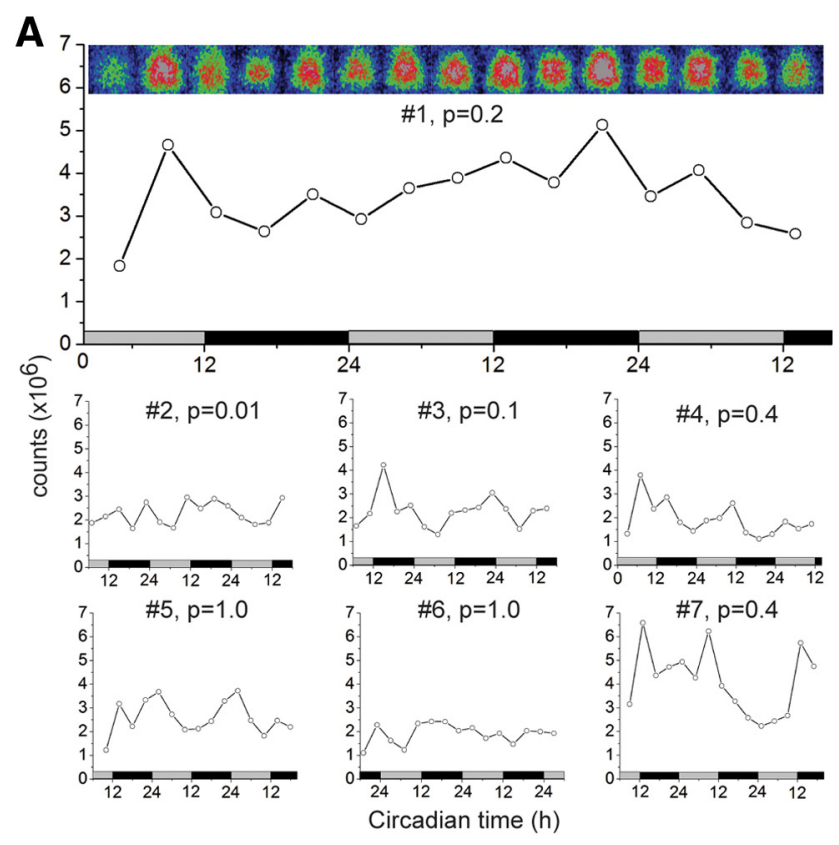

B

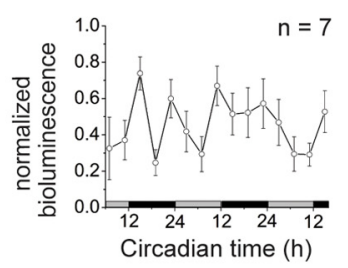

Figure 4. Circadian rhythmicity in the $O B$ requires VIP. $A$, After 2 weeks in DD, PER2::LUC bioluminescence from the $O B$ (pseudocolored for mouse 1) was arrhythmic in six of seven $\mathrm{Vip}^{-1-}$ mice. $\boldsymbol{B}$, Bioluminescence was normalized in individual mice and then averaged across mice. Mice were maintained under DD for 2 weeks so they were on different CTs during imaging sessions. Each time point was pooled within a $2 \mathrm{~h}$ window. Mean bioluminescence did not significantly differ across CT ( $n=7$ mice). Gray and black bars denote subjective day (CT 0 -CT 12) and night (CT 12-CT 24), respectively. Counts are the mean number of photons integrated over $10 \mathrm{~min}$.

for sustained circadian rhythms in the $\mathrm{OB}$ in the absence of external timing cues.

\section{VIP is required for a circadian rhythm in odor} detection performance

To test whether VIP is also required for circadian rhythms in olfactory-dependent behavior, we tested olfactory perception of VIP-deficient, VIP-receptor deficient, and WT mice. Mice were trained to sniff and indicate if they detected vanilla odor. After

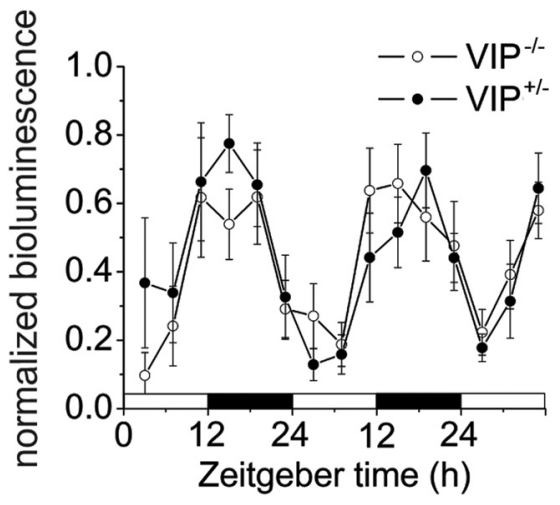

Figure 5. Both $\mathrm{Vip}^{+/-}$and $\mathrm{Vip}^{-/-}$mice exhibited a circadian rhythm in PER2:::LUC bioluminescence in vivo in a light/dark cycle. Gray bars denote the times of lights on and black bars the times of darkness. Bioluminescence was normalized (minimum is 0 and maximum is 1 ) in individual mice and then averaged across mice of the same genotype. ZT 0 and ZT 24 denote 7:00 A.M., and ZT 12 denotes 7:00 P.M. in a light/dark cycle. Mean \pm SEM.

reaching a criterion of $85 \%$ correct responses at approximately $17 \mathrm{~d}$ after training started, mice were switched to DD for $2 \mathrm{~d}$ and tested every 8 h over 2 consecutive days. We found no differences in the number of days required to reach the criterion between three genotypes, suggesting that learning was not impaired in $\operatorname{Vip}^{-1-}$ and Vipr2 $2^{-/-}$mice $\left(F_{(2,17)}=0.3 ; p=0.7\right.$, one-way ANOVA with a Tukey's post hoc test). Compared with WT mice, $V i p r 2^{-1-}$ and $V i p^{-/-}$mice spent more time sniffing the odorant port at all times of day $\left(F_{(3,1468)}=92.4 ; p<0.01\right.$ for all CTs, one-way ANOVA with a Tukey's post hoc test).

WT mice showed statistically significant daily variations in their odor detection performance, peaking at approximately CT 16 -CT 20 (Fig. $6 ; F_{(5,71)}=2.7 ; p<0.05,0.001,0.05,0.005$, and 0.01 for $1: 10^{1}$ through $1: 10^{5}$ dilutions, respectively, two-way ANOVA with a Tukey's post hoc test), whereas $V_{i p}{ }^{-1-}$ or $V_{\text {ipr }} 2^{-1-}$ mice did not show daily variations in their odor detection performance $\left(F_{(5,71)}=0.7 ; p=0.07\right.$, two-way ANOVA with a Tukey's post hoc test). Interestingly, compared with WT mice, $V_{i p}{ }^{-/-}$mice performed better during the subjective day at all vanilla dilutions $\left(F_{(2,25)}=2 ; p<0.01\right.$, one-way ANOVA with a Tukey's post hoc test), whereas Vipr2 ${ }^{-1-}$ mice performed, on average, similarly as WT mice at all times for all vanilla dilutions $\left(F_{(2,25)}=1.9 ; p=0.06\right.$, one-way ANOVA with a Tukey's post hoc test; see Discussion). Although there was a statistically significant difference in the performance during the subjective day between WT and Vip ${ }^{-1-}$ mice, Vip ${ }^{-1-}$ and Vipr $2^{-1-}$ mice performed, on average, in a similar manner $\left(F_{(2,25)}=1.9 ; p=0.06\right.$, one-way ANOVA with a Tukey's post hoc test). These results indicate that VIP or its receptor is required for daily regulation of olfactory sensitivity.

\section{Discussion}

A circadian rhythm in the $\mathrm{OB}$ correlates with changes in olfactory performance

Previous work showed that a circadian rhythm in olfaction depends on canonical clock genes (Granados-Fuentes et al., 2011), but the underlying cells and circuits are essentially unknown. Here, we demonstrated intrinsic circadian rhythmicity in the isolated $\mathrm{OE}$ and $\mathrm{OB}$ but not in the isolated $\mathrm{PC}$, suggesting that the early stages in the olfactory circuit may contribute a circadian rhythmicity in olfaction. This is consistent with previous evidence that circadian rhythms in olfaction do not require the SCN (Granados-Fuentes et al., 2011) and that circadian rhythms of the 


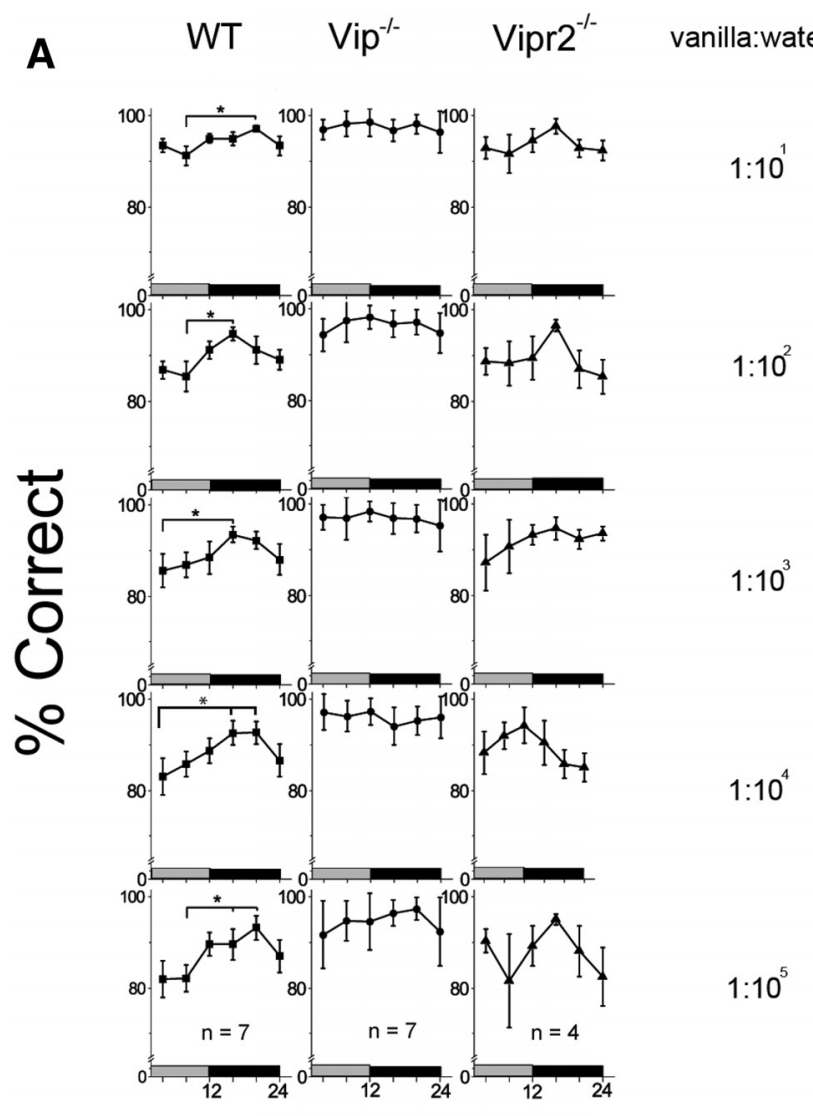

B

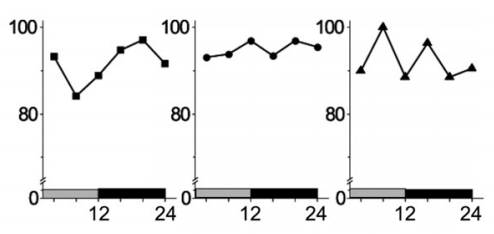

$1: 10^{1}$

\section{Circadian time $(\mathrm{h})$}

Figure 6. Circadian rhythms in odor detection were blunted in Vip ${ }^{-/-}$and Vipr2 ${ }^{-/-}$compared with WT mice after $48 \mathrm{~h}$ in DD. WT mice showed a peak during the subjective night in performance at all five concentrations of vanilla tested $(\boldsymbol{A})$. Representative mice in each group showing odor detection performance at 1:10 ${ }^{1}$ vanilla dilution $(\boldsymbol{B})$. The gray and black bars indicate subjective day and night, respectively. Mean \pm SEM. $n$ is number of mice per group.

odor-evoked c-fos expression in the PC do require input from the ipsilateral OB (Granados-Fuentes et al., 2006). Furthermore, the times of peak PER2 expression in the OB (CT 18-CT 22) correlate with the nighttime peak in olfactory performance (CT 16-CT 20). Interestingly, the circadian period of the isolated OE was, on average, $5 \mathrm{~h}$ shorter than that of the OB. It will be interesting to test whether this in vitro difference in circadian period of the $\mathrm{OE}$ and $\mathrm{OB}$ have any effect on their in vivo phase relationships as has been found for the different times of peak activity in the $\mathrm{OB}$ and SCN (Granados-Fuentes et al., 2004a). These results implicate circadian pacemaking cells in the OB in daily modulation of odor detection.

VIP plays a conserved role in coordinating circadian rhythms in the SCN and OB

VIP is expressed in neurons of the SCN and OB, and loss of VIP signaling diminishes synchronized circadian oscillations among

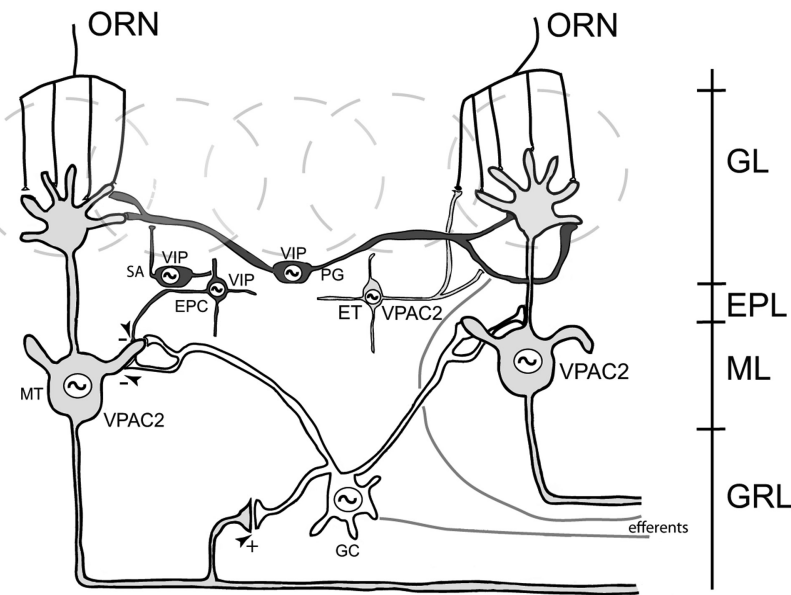

Figure 7. Proposed circuit for circadian modulation of olfactory sensitivity. Within the $O B$, circadian variations in VIP release from a subset of interneurons regulate excitability of MT and ET cells by acting on VPAC2R expressed on their dendrites and cell bodies. EPC, external plexiform layer cell; GC, granular cell; GL, glomerular layer; GRL, granular layer; ML, mitral cell layer; $\mathrm{ORN}$, olfactory receptor neuron; $\mathrm{PG}$, periglomerular cell; $\mathrm{SA}$, short axon cell. Arrowhead with -, Inhibitory; arrowhead with + , excitatory.

SCN cells and coordinated circadian locomotor behavior (Aton and Herzog, 2005; Aton et al., 2005). Therefore, we postulated that VIP signaling may play a similar role in the OB and in olfaction. We found that VIP is required for synchronizing the circadian rhythm in PER2 expression of the $\mathrm{OB}$ in vivo and in odor detection performance. Furthermore, olfactory performance and PER2 expression in the OB, like locomotor activity, of VIPdeficient mice remain rhythmic in a light/dark cycle and gradually becomes arrhythmic in constant conditions.

In addition to the loss of the circadian rhythm in odor detection performance, Vip $^{-1-}$ mice performed better than WT mice during the subjective day at all concentrations of the odor, whereas Vipr $2^{-1-}$ mice were similar to the average daily performance of WT mice. Although both VIP and VPAC2R are strongly expressed in the OB, VIP and VPAC2R are also differentially expressed in the other brain areas (www.gensat.org; www. brain-map.org). Therefore, we cannot rule out the possibility that loss of VIP versus VPAC2R in the other brain areas could differentially influence the behavioral performance in $V_{i p}{ }^{-1-}$ versus Vipr $2^{-1-}$ mice. For example, it is possible that loss of VIP in the VIP-expressing interneurons of the PC could influence odor-evoked activity of pyramidal neurons and thereby affect olfactory perception. However, the fact that $\mathrm{Vip}^{-/-}$mice exhibit arrhythmicity in PER2 expression in the OB and both Vip ${ }^{-/-}$and Vipr $2^{-1-}$ mice exhibit arrhythmicity in odor detection performance suggests that the loss of Vip or Vipr2 in the OB results in the loss of the circadian rhythm in olfactory-dependent behavior.

We also found evidence that VIP abundance in the OB peaks during the night. This could reflect a daily rhythm in release and/or synthesis of VIP. VIP release from the SCN is circadian in vitro and peaks early in the day in vivo (Laemle et al., 1995; Shinohara et al., 1995, 1998; Honma et al., 1998; Francl et al., 2010). Together, these results suggest that VIP plays a conserved role in maintaining and synchronizing circadian rhythms in both the SCN and OB.

\section{A proposed circuit for circadian modulation of olfactory processing}

VIPergic interneurons in the $\mathrm{OB}$ appear to be well positioned to modulate the output of the OB. We found that VIPergic in- 
terneurons are concentrated in the PGL and EPL. The processes of these VIPergic interneurons cover the entire EPL and regions of the glomerular layer. Consistent with the distribution of VIPergic terminals, we found that VPAC2Rs are expressed in most, if not all, cell bodies and dendritic fields of MT/ET cells. Furthermore, VIP levels in the EPL interneurons peak during the evening, suggesting daily variation in VIP release. Therefore, we postulate that daily rhythms in VIP release from a subset of EPL cells and probably also PGL cells modulate the activity of MT/ET cells, the projection neurons of the $\mathrm{OB}$ (Fig. 7).

The $\mathrm{OB}$, but not the PC, was shown to be intrinsically circadian in vitro (Granados-Fuentes et al., 2006), and yet both MT/ET cells and cells of the PC exhibit greater odor-evoked c-fos expression during the night in vivo (Granados-Fuentes et al., 2006). Therefore, we postulate that VIP signaling onto MT/ET cells likely augments the sensitivity of MT/ET cells at night and, indirectly, their targets in the PC and other higher brain areas processing olfactory information. This daily rhythm may ensure sufficient sensitivity at night when mice forage for food and are vulnerable to predators.

\section{References}

Abraham U, Prior JL, Granados-Fuentes D, Piwnica-Worms DR, Herzog ED (2005) Independent circadian oscillations of Period1 in specific brain areas in vivo and in vitro. J Neurosci 25:8620-8626. CrossRef Medline

Aton SJ, Herzog ED (2005) Come together, right. . . now: synchronization of rhythms in a mammalian circadian clock. Neuron 48:531-534. CrossRef Medline

Aton SJ, Colwell CS, Harmar AJ, Waschek J, Herzog ED (2005) Vasoactive intestinal polypeptide mediates circadian rhythmicity and synchrony in mammalian clock neurons. Nat Neurosci 8:476-483. CrossRef Medline

Brand C, Burkhardt E, Schaeffel F, Choi JW, Feldkaemper MP (2005) Regulation of Egr-1, VIP, and Shh mRNA and Egr-1 protein in the mouse retina by light and image quality. Mol Vis 11:309-320. Medline

Brown TM, Hughes AT, Piggins HD (2005) Gastrin-releasing peptide promotes suprachiasmatic nuclei cellular rhythmicity in the absence of vasoactive intestinal polypeptide-VPAC2 receptor signaling. J Neurosci 25: 11155-11164. CrossRef Medline

Colwell CS, Michel S, Itri J, Rodriguez W, Tam J, Lelievre V, Hu Z, Liu X, Waschek JA (2003) Disrupted circadian rhythms in VIP- and PHIdeficient mice. Am J Physiol Regul Integr Comp Physiol 285:R939-R949. CrossRef Medline

Crespo C, Blasco-Ibáñez JM, Marqués-Marí AI, Alonso JR, Briñón JG, MartínezGuijarro FJ (2002) Vasoactive intestinal polypeptide-containing elements in the olfactory bulb of the hedgehog (Erinaceus europaeus). J Chem Neuroanat 24:49-63. CrossRef Medline

Dibner C, Schibler U, Albrecht U (2010) The mammalian circadian timing system: organization and coordination of central and peripheral clocks. Annu Rev Physiol 72:517-549. CrossRef Medline

Francl JM, Kaur G, Glass JD (2010) Regulation of vasoactive intestinal polypeptide release in the suprachiasmatic nucleus circadian clock. Neuroreport 21:1055-1059. CrossRef Medline

Gracia-Llanes FJ, Crespo C, Blasco-Ibáñez JM, Marqués-Marí AI, MartínezGuijarro FJ (2003) VIP-containing deep short-axon cells of the olfactory bulb innervate interneurons different from granule cells. Eur J Neurosci 18:1751-1763. CrossRef Medline

Granados-Fuentes D, Prolo LM, Abraham U, Herzog ED (2004a) The suprachiasmatic nucleus entrains, but does not sustain, circadian rhythmicity in the olfactory bulb. J Neurosci 24:615-619. CrossRef Medline
Granados-Fuentes D, Saxena MT, Prolo LM, Aton SJ, Herzog ED (2004b) Olfactory bulb neurons express functional, entrainable circadian rhythms. Eur J Neurosci 19:898-906. CrossRef Medline

Granados-Fuentes D, Tseng A, Herzog ED (2006) A circadian clock in the olfactory bulb controls olfactory responsivity. J Neurosci 26:1221912225. CrossRef Medline

Granados-Fuentes D, Ben-Josef G, Perry G, Wilson DA, Sullivan-Wilson A, Herzog ED (2011) Daily rhythms in olfactory discrimination depend on clock genes but not the suprachiasmatic nucleus. J Biol Rhythms 26:552560. CrossRef Medline

Harmar AJ, Marston HM, Shen S, Spratt C, West KM, Sheward WJ, Morrison CF, Dorin JR, Piggins HD, Reubi JC, Kelly JS, Maywood ES, Hastings MH (2002) The VPAC(2) receptor is essential for circadian function in the mouse suprachiasmatic nuclei. Cell 109:497-508. CrossRef Medline

Honma S, Katsuno Y, Tanahashi Y, Abe H, Honma K (1998) Circadian rhythms of arginine-vasopressin and vasoactive intestinal polypeptide do not depend on cytoarchitecture of dispersed cell culture rat suprachiasmatic nucleus. Neuroscience 86:967-976. CrossRef Medline

Hu E, Demmou L, Cauli B, Gallopin T, Geoffroy H, Harris-Warrick RM, Paupardin-Tritsch D, Lambolez B, Vincent P, Hepp R (2011) VIP, CRF, and PACAP act at distinct receptors to elicit different cAMP/PKA dynamics in the neocortex. Cereb Cortex 21:708-718. CrossRef Medline

Hughes ME, Hogenesch JB, Kornacker K (2010) JTK_CYCLE: an efficient nonparametric algorithm for detecting rhythmic components in genome-scale data sets. J Biol Rhythms 25:372-380. CrossRef Medline

Laemle LK, Ottenweller JE, Fugaro C (1995) Diurnal variations in vasoactive intestinal polypeptide-like immunoreactivity in the suprachiasmatic nucleus of congenitally anophthalmic mice. Brain Res 688:203-208. CrossRef Medline

Maywood ES, Reddy AB, Wong GK, O’Neill JS, O’Brien JA, McMahon DG, Harmar AJ, Okamura H, Hastings MH (2006) Synchronization and maintenance of timekeeping in suprachiasmatic circadian clock cells by neuropeptidergic signaling. Curr Biol 16:599-605. CrossRef Medline

Nakamachi T, Matkovits A, Seki T, Shioda S (2012) Distribution and protective function of pituitary adenylate cyclase-activating polypeptide in the retina. Front Endocrinol (Lausanne) 3:145-155. CrossRef Medline

Sanides-Kohlrausch C, Wahle P (1990) VIP- and PHI-immunoreactivity in olfactory centers of the adult cat. J Comp Neurol 294:325-339. Medline

Shinohara K, Honma S, Katsuno Y, Abe H, Honma K (1994) Circadian rhythms in the release of vasoactive intestinal polypeptide and argininevasopressin in organotypic slice culture of rat suprachiasmatic nucleus. Neurosci Lett 170:183-186. CrossRef Medline

Shinohara K, Honma S, Katsuno Y, Abe H, Honma K (1995) Two distinct oscillators in the rat suprachiasmatic nucleus in vitro. Proc Natl Acad Sci U S A 92:7396-7400. CrossRef Medline

Shinohara K, Tominaga K, Inouye ST (1998) Luminance-dependent decrease in vasoactive intestinal polypeptide in the rat suprachiasmatic nucleus. Neurosci Lett 251:21-24. CrossRef Medline

Slotnick B, Restrepo D (2005) Olfactometry with mice. Curr Protoc Neurosci Chapter 8:Unit 8.20. CrossRef Medline

Vosko AM, Schroeder A, Loh DH, Colwell CS (2007) Vasoactive intestinal peptide and the mammalian circadian system. Gen Comp Endocrinol 152:165-175. CrossRef Medline

Welsh DK, Takahashi JS, Kay SA (2010) Suprachiasmatic nucleus: cell autonomy and network properties. Annu Rev Physiol 72:551-577. CrossRef Medline

Zou YC, Liu LQ, Zhang MX (2014) The expression of vasoactive intestinal polypeptide in visual cortex-17 in normal visual development and formation of anisometropic amblyopia. Semin Ophthalmol 29:59-65. CrossRef Medline 